\title{
Estimation of Single Brainstem Auditory Evoked Potential Using Time-Sequenced Adaptive Filtering
}

\author{
F.H.Y.Chan, F.K.Lam, P.W.F.Poon* \\ Department of Electrical \& Electronic Engineering, *Department of Physiology \\ The University of Hong Kong, \\ Pokfulam Road, Hong Kong \\ W.Qiu and B.Z.Xu \\ Institute of Radio Engineering and Automation \\ South China University of Technology, \\ Guangzhou, 510641, P.R.China
}

\begin{abstract}
Time-sequenced adaptive filtering (TSAF) technique has been successfully used to track variation of brainstem auditory evoked potential (BAEP) in human. 400 ensembles used by TSAF will produce a satisfactory result rivaling to EA using 2000 ensembles. Furthermore, single BAEP signal can be obtained which make it possible for clinicians to analyze the variation of signals across trials.
\end{abstract}

Keywords: Time-Sequenced Adaptive Filter, Brainstem auditory evoked potential, Ensemble averaging.

\section{INTRODUCTION}

Evoked potentials (EPs), which are brain electrical activities resulting from sensory stimulation, are time-locked signals. Ensemble averaging (EA) is the most widely used method for obtaining the EPs. However, this method ignores the fact that the EPs are also time-varying signals and the results of EA may introduce significant distortion and loss of information about the response variability from trial to trial. In this paper, we present an adaptive filtering method based on [1] to track variation of brainstem auditory evoked potential (BAEP) and improve the SNR.

\section{METHOD}

Fig. 1 shows a conceptual realization of TSAF. Differing from conventional adaptive signal enhancer, the TSAF uses multiple filters and each filter is adapted for filtering a particular portion of the interval between regeneration times, and is suitable for tracking signals whose statistical properties recur at various points in time. The filters are trained and the filter weights are obtained via an adaptive algorithm.

The TSAF filter has been applied to detect BAEP in humans. The stimulus pulse was of $0.1 \mathrm{msec}$ duration and the click rate was $10 \mathrm{~Hz}$. The $100 \mathrm{~dB}$ sound pressure level (SPL) was given to a subject. The response was recorded at a sampling rate of $10 \mathrm{kHz}$. The first $9 \mathrm{~ms}$ of response has been processed for BAEP evaluation. The SNR of the raw data of human is about $-20 \mathrm{~dB}$ in our case. The regeneration time were set at the instant of stimuli were given. The sequence number was initialized at the regeneration time and then incremented after every point until 90 set of weights have been cycled through. Each set contains 25 weights.

\section{RESULTS AND CONCLUSIONS}

Using TSAF we can track the variation across trials after convergence. Fig. 2 shows two results of single BAEP in human estimated by TSAF. The SNR of single trial was be significantly improved and the five peaks are clear. Fig. 3 gave the trace of BAEP of 600 trials. Since the result of EA is still the standard of studying BAEP in clinical application, it is necessary to make a comparison the result of averaged TSAF with that of EA. We use 400 trials to estimate an averaged result of TSAF. First 200 trials are used for training the filters and last 200 trials processed by TSAF are averaged to get an averaged estimate of BAEP. The averaged TSAF is shown in Fig. 4 together the EA using 2000 trials. TSAF and EA are very similar and their correlation coeflicient is 0.983 , and the measurement time greatly reduced using TSAF. The tracking ability of TSAF makes it possible for the clinician to observe the signal variation trace in every single ensemble which may be helpful for the detail analysis of EPs.

\section{ACKNOWLEDGMENTS}

This project has been supported in part by University of Hong Kong Research Grants. 


\section{REFERENCES}

[1] E.R.Ferrara and B.Widrow, "The time-sequenced adaptive filter", IEEE trans. Acoust., Speech, Signal Processing, vol. ASSP-29, pp. 679-683., 1981.

[2] P.Laguna, O.Meste et al, "Adaptive Filter for EventRelated Bioelectric Signals Using an Impulse Correlated Reference Input: Comparison with Signal Averaging Techniques", IEEE Trans. Biomed. Eng., vol. 39, pp. 1032-1044, 1992.

[3] F.K.Lam, F.H.Y.Chan, W.Qiu and P.W.F.Poon, "Adaptive Processing of Early Evoked Potentials", Australian Conference on Physical Science and Eng. in Medicine and Biomedical Eng., pp. 115, Sept., 1993.

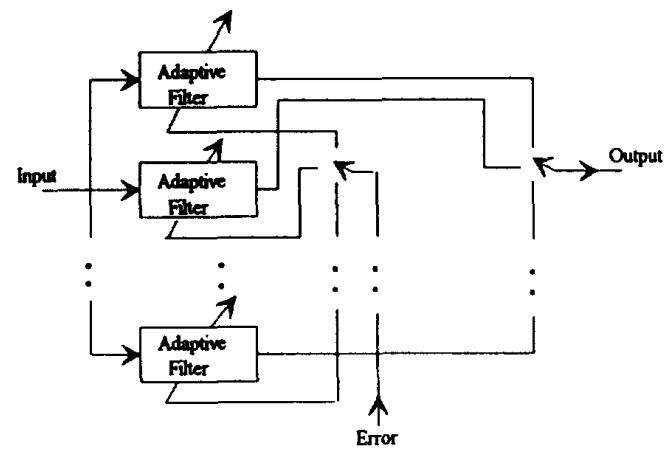

Fig. 1 The time-sequences adaptive filter

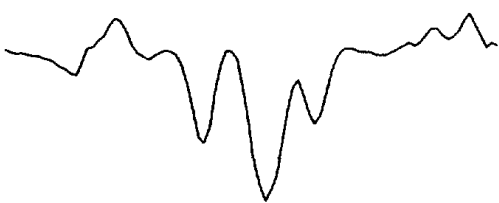

ms

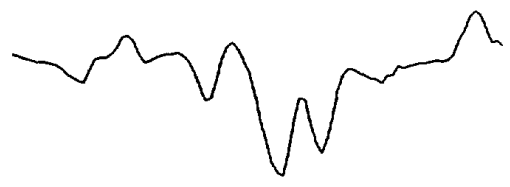

ms

Fig. 2 Two individual BAEP waveforms estimated by TSAF

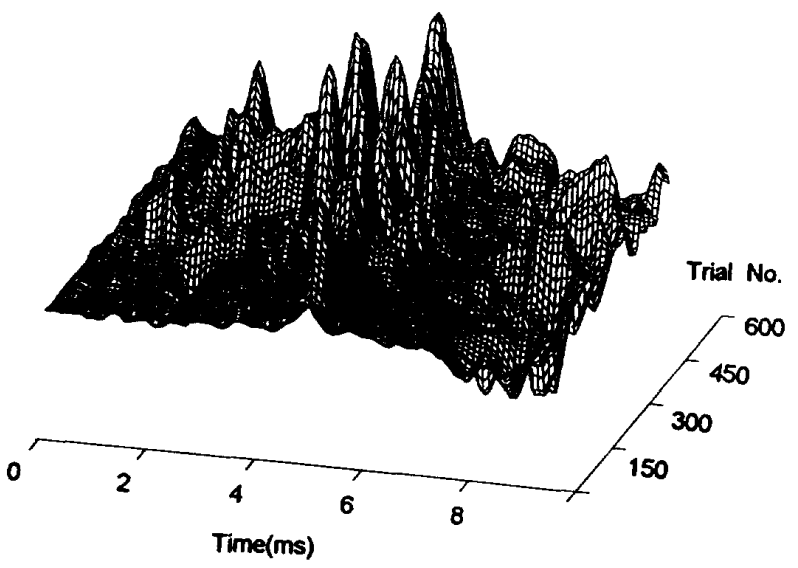

(a)

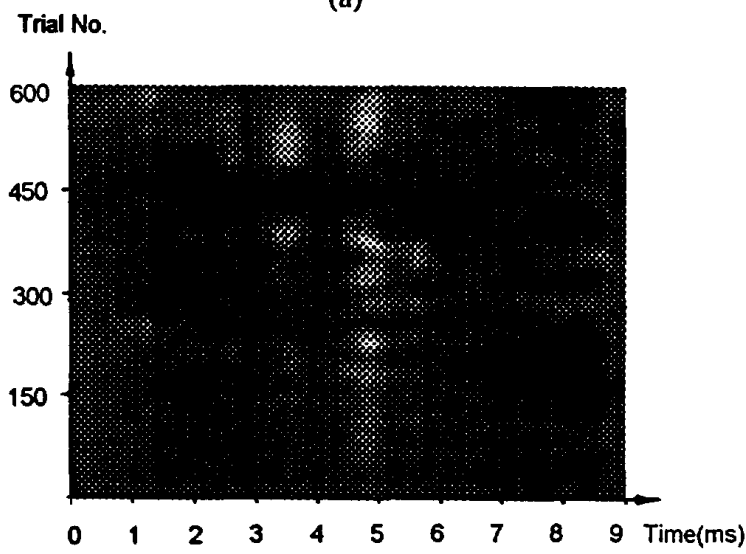

(b)

Fig. 3 Trace of BAEP of 600 trials. (a) a 3D view of BAEP trace. (b) a gray level 2D view of (a).

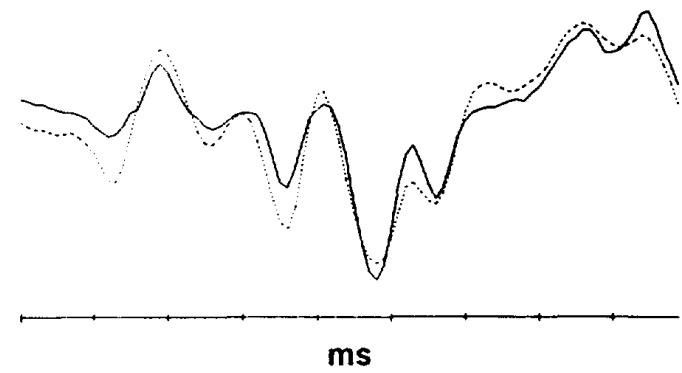

Fig. 4 A comparison of averaged TSAF (solid line) and EA (dotted line) 